\title{
Imaging the Surface of Altair
}

\author{
John D. Monnier ${ }^{1 *}$, M. Zhao ${ }^{1}$, E. Pedretti ${ }^{2}$, N. Thureau ${ }^{3}$, M. Ireland ${ }^{4}$, \\ P. Muirhead ${ }^{5}$, J.-P. Berger ${ }^{6}$, R. Millan-Gabet ${ }^{7}$, \\ G. Van Belle ${ }^{7}$, T. ten Brummelaar ${ }^{8}$, H. McAlister ${ }^{8}$, S. Ridgway ${ }^{9}$, \\ N. Turner ${ }^{8}$, L. Sturmann ${ }^{8}$, J. Sturmann ${ }^{8}$, D. Berger ${ }^{1}$ \\ ${ }^{1}$ Department of Astronomy, University of Michigan, \\ ${ }^{2}$ University of St. Andrews, Scotland, UK \\ ${ }^{3}$ University of Cambridge, Cambridge, UK \\ ${ }^{4}$ California Institute of Technology, Pasadena, CA \\ ${ }^{5}$ Cornell University, Ithaca, NY \\ ${ }^{6}$ Laboratoire d'Astrophysique de Grenoble, France \\ ${ }^{7}$ Michelson Science Center, Pasadena, CA \\ ${ }^{8}$ CHARA, Georgia State University, Atlanta, GA \\ ${ }^{9}$ National Optical Astronomy Observatory, Tucson, AZ \\ *To whom correspondence should be addressed; E-mail: monnier@umich.edu.
}

Spatially resolving the surfaces of nearby stars promises to advance our knowledge of stellar physics. Using optical long-baseline interferometry, we present here a near-infrared image of the rapidly rotating hot star Altair with $<1$ milliarcsecond resolution. The image clearly reveals the strong effect of gravity darkening on the highly-distorted stellar photosphere. Standard models for a uniformly rotating star can not explain our results, requiring differential rotation, alternative gravity darkening laws, or both.

While solar astronomers can take advantage of high-resolution, multi-wavelength, real-time imaging of the Sun's surface, stellar astronomers know most stars, located parsecs or kilopar- 
secs away, as simple points of light. To discover and understand the novel processes around stars unlike the Sun, we must rely on stellar spectra averaged over the entire photosphere. Despite their enormous value, spectra alone have been inadequate to resolve central questions in stellar astronomy, such as the role of angular momentum in stellar evolution (1), the production and maintenance of magnetic fields (2), the launching of massive stellar winds (3), and the interactions between very close binary companions (4).

Fortunately, solar astronomers no longer hold a monopoly on stellar imaging. Using longbaseline visible and infrared interferometers, the photospheric diameters of hundreds of stars and high precision dynamical masses for dozens of binaries have been catalogued, offering exacting constraints for theories of stellar evolution and stellar atmospheres (5). This work requires an angular resolution of $\sim 1$ milliarcseconds ( 1 part in $2 \times 10^{8}$, or 5 nano-radians) for resolving even nearby stars and is more than an order-of-magnitude better than that achievable with the Hubble Space Telescope or ground-based 8-m class telescopes equipped with adaptive optics.

Stellar imaging can be used to investigate rapid rotation of hot, massive stars. A significant fraction of hot stars are rapid rotators with surface rotational velocities of more than 100 $\mathrm{km} / \mathrm{s}(6.7)$. These rapid rotators are expected to traverse very different evolutionary paths than their slowly rotating kin (1) and rotation-induced mixing alters stellar abundances (8). While hot stars are relatively rare by number in the Milky Way Galaxy, they have a disproportional effect on galactic evolution due to their high luminosities, strong winds, and their final end as supernovae (for the most massive stars). Recently, rapid rotation in single stars has been invoked to explain at least one major type of gamma ray bursts $(9)$ and binary coalescence of massive stars/remnants for another (10).

The distinctive observational signatures of rapid rotation were first described by von Zeipel (11), beginning with the expection that centrifugal forces would distort the photospheric shape 
and that the resulting oblateness would induce lower effective temperatures at the equator. This latter effect, known as gravity darkening, will cause distortions in the observed line profiles as well as the overall spectral energy distribution. Precise predictions can be made but rely on uncertain assumptions, most critically the distribution of angular momentum in the star uniform rotation is often assumed for simplicity.

The most basic predictions of von Zeipel theory - centrifugal distortion and gravity darkening - have been confirmed to some extent. The Palomar Testbed Interferometer (PTI) was first to measure photospheric elongation in a rapid rotator, finding the diameter of the nearby A-type star Altair to be $\sim 14 \%$ larger in one dimension than the other (12). The Navy Prototype Optical Interferometer (NPOI) and the Center For High Angular Resolution Astronomy (CHARA) interferometric array both measured strong limb-darkening profiles for the photometric standard Vega (13 14), consistent with rapid rotator viewed nearly pole-on. A brightness asymmetry for Altair was also reported by NPOI (15, 16), suggestive of the expected pole-to-equator temperature difference from gravity darkening. In recent years, a total of five rapid rotators have been measured to be elongated by interferometers (17, 18, 19).

While von Zeipel theory appears to work at a basic level, serious discrepancies between theory and observations have emerged. Most notably, the diameter of the B3V-type star Achernar (17) was measured to be $\sim 1.56$ times longer in one dimension than the other, too large to be explained by von Zeipel theory. Explanations for this include strong differential rotation of the star (20) or the presence of a polar wind (3), either of which have far-reaching consequences for our understanding of stellar evolution. In order to address these issues, we must move beyond the simplest models for rapidly rotating stars, and this will require a corresponding jump in the quality and quantity of interferometry data. Indeed, all previous results were based on limited interferometer baselines, lacking the capability to form model-independent images, relying entirely on model-fitting for interpretation. Thus previous confirmations of von Zeipel theory, 
although suggestive, were incomplete.

Here we report a development in imaging capabilities that tests the von Zeipel theory, both through basic imaging and precise model-fitting. By combining near-infrared light from four telescopes of the Georgia State University CHARA interferometric array, we have synthesized an elliptical aperture with dimensions $265 \times 195$ meters (Figure 1), allowing us to reconstruct images of the prototypical rapid rotator Altair (spectral type A7V) with an angular resolution of $\sim 0.64$ milliarcseconds, the diffraction limit defined by $\frac{\lambda}{2 D}$, the observing wavelength divided by twice the longest interferometer baseline. The recently-commissioned Michigan Infrared Combiner (MIRC) (2I) was essential for this work, allowing the light from the CHARA telescopes to be all combined together simultaneously in 8 spectral channels spanning the astronomical $\mathrm{H}$ band $(\lambda=1.50-1.74 \mu \mathrm{m})$. The Altair data presented here were collected on UT2006Aug31 and UT2006Sep01 - complete observational information is available (22). In addition, we utilized some $\mathrm{K}$ band $(\lambda=2.2 \mu \mathrm{m})$ observations by the PTI to constrain the short-baseline visibilities in subsequent analysis.

By using four CHARA telescopes, interferometric imaging of Altair is now possible, although this requires specialized image reconstruction techniques. We utilized the publiclyavailable application "Markov-Chain Imager for Optical Interferometry (MACIM)" (23) in this work, applying the Maximum Entropy Method (MEM) (24). We restricted the stellar image to fall within an elliptical boundary, similar in principle to limiting the field-of-view in standard aperture synthesis procedures. This restriction biases our imaging against faint emission features arising outside the photosphere; however, we do not expect any circumstellar emission in Altair which is relatively cool, lacking signs of gas emission or strong winds. Further details of our imaging procedures, along with results from validation tests, can be found in (22).

Our image shows the stellar photosphere of Altair to be well-resolved (Figure 2A), appearing elongated in the NE-SW direction with a bright dominant feature covering the northwest 
quadrant of the star. In order to reduce the influence of possible low-level artifacts that are beyond the diffraction-limit of our interferometer, we have followed the standard procedure (25) of convolving the reconstructed image with a Gaussian beam matching the resolution of the interferometer (Figure 2B).

These images confirm the basic picture of gravity darkening induced by rapid rotation. We see Altair's photosphere to be oblate with a bright region identifiable as the stellar polar region. The intensity of the dark equatorial band is approximately $60-70 \%$ of the brightness at the pole, broadly consistent with expectations for the near-infrared from previous models. While we see some evidence for deviations from axisymmetry (small excess emission on northern limb), this feature is at the limit of our image fidelity and will require additional Fourier coverage to investigate further.

We have also fitted our new extensive dataset with a rapid rotator model, following the prescription set out in Aufdenberg et al. (14) and references therein, assuming a Roche potential (central point mass) and solid body rotation. The main parameters of the model are the stellar radius and temperature at the pole, the angular rotation rate as a fraction of breakup $(\omega)$, the gravity darkening coefficient $(\beta)$ and the viewing angles (inclination and position angle). We employed the stellar atmosphere models of Kurucz (26) for determining the specific intensity of each point on the surface as a function of local gravity, effective temperature, and limb darkening. In addition to matching the new MIRC/CHARA data, we forced the model to match the measured $\mathrm{V}$ and $\mathrm{H}$ band photometric magnitudes $(0.765 \pm 0.015$ and $0.235 \pm 0.043$ respectively) derived from a broad literature survey. When fixing the gravity-darkening cofficient to $\beta=0.25$ appropriate for radiative envelopes, our derived parameters (Table 1) agree well with best-fit parameters of Peterson et al. (15) based on visible data. However, our best-fit model reached only a reduced $\chi_{\nu}^{2}$ of 1.79 , suggesting a need for additional degrees of freedom in our model. 
In order to improve our fits, we explored an extension to the von Zeipel model, allowing the gravity darkening coefficient $\beta$ to be a free parameter. We found that $\beta=0.190$ model significantly improved the goodness-of-fit (Table 1) and this improvement is visually apparent when comparing synthetic model images to the Altair image from CHARA (Figure 3). In addition to a lower $\beta$, the new model prefers a slightly less inclined orientation, a cooler polar temperature, and a faster rotation rate.

Both our imaging and modeling results point to important deficiencies in the currentlypopular models for rapid rotators. Previous workers have also encountered problems explaining high-resolution interferometry data with standard prescriptions for rotating stars. In addition to the Achernar case previously cited, Peterson et al. (15) were unable to find a satisfactory fit for Altair assuming a standard Roche - von Zeipel model $\left(\chi_{\nu}^{2}=3.8\right)$, consistent with the need for additional stellar physics. Recent results for Alderamin (19) also specifically favor models with smaller $\beta$ s, in line with our findings.

While model fitting has revealed deviations from standard theory, our model-independent imaging allows new features to be discovered outside current model paradigms. The most striking difference between our CHARA image and the synthetic model images (Figure 3) is that our image shows stronger darkening along the equator, inconsistent with any von Zeipellike gravity darkening prescription assuming uniform rotation.

Lower equatorial surface temperatures could naturally arise if the equatorial rotation rate was higher than the rest of the star (differential rotation), reducing the effective gravity at the surface (27). Another possibility is that the cooler equatorial layers could be unstable to convection (28, 29), invalidating a single gravity darkening "law" applicable to all stellar latitudes. Other studies (30) have found further faults with simple application of the von Zeipel law due to opacity effects in the surface layers.

While difficult to isolate or untangle these various effects from one another, nevertheless 
the new interferometric results and our modeling convincingly establishes the case for stellar physics beyond the standard models used today to describe rotating stars. A path forward is clear: differential rotation will leave both geometric and kinematic signatures different from opacity or convection-related phenomena. Observers must be armed with a new generation of models incorporating these physical processes in order to exploit the powerful combination of detailed line profile analysis and multi-wavelength interferometric imaging now available. 


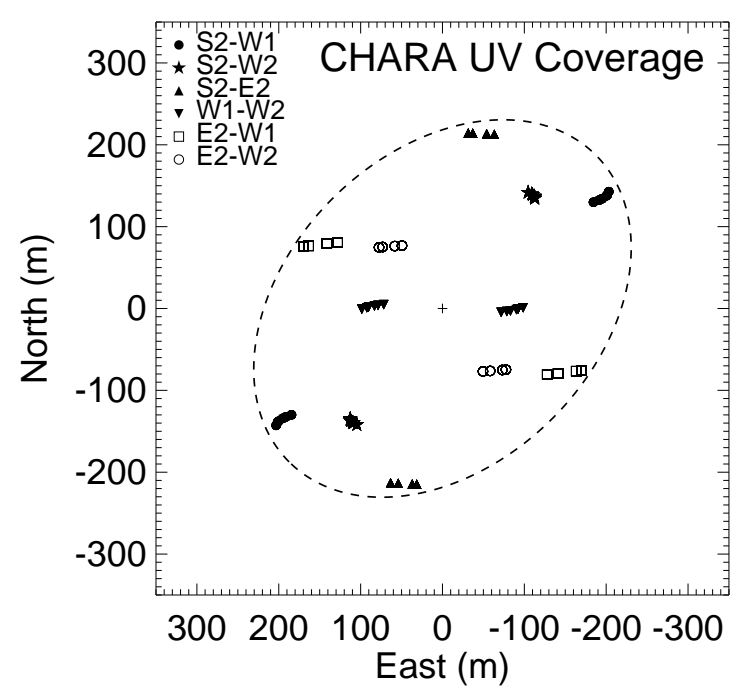

Figure 1: This figure shows the Fourier UV coverage for the Altair observations, where each point represents the projected separation between one pair of CHARA telescopes (S2-E2-W1-W2) (31). The dashed ellipse shows the equivalent coverage for an elliptical aperture of $265 \times 195$ meters oriented along a Position Angle of $135^{\circ}$ East of North. 

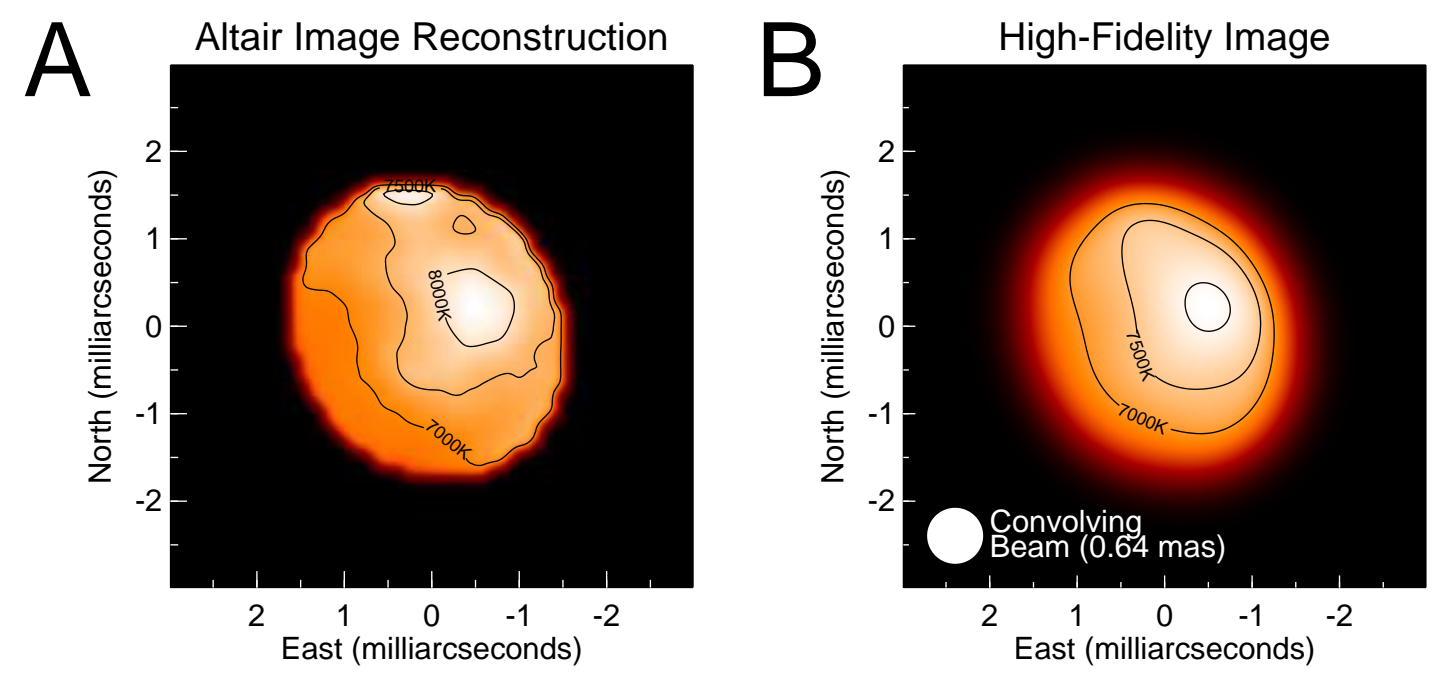

Figure 2: A) shows the intensity image of the surface of Altair $(\lambda=1.65 \mu \mathrm{m})$ created with the MACIM/MEM imaging method using a uniform brightness elliptical prior $\left(\chi_{\nu}^{2}=0.98\right)$. Typical photometric errors in the image correspond to $\pm 4 \%$ in intensity. B) shows the reconstructed image convolved with a Gaussian beam of 0.64 mas, corresponding to the diffraction-limit of CHARA for these observations. For both panels, the specific intensities at $1.65 \mu \mathrm{m}$ were converted into the corresponding blackbody temperatures and contours for $7000 \mathrm{~K}, 7500 \mathrm{~K}$, and $8000 \mathrm{~K}$ are shown. North is up and East is left. 

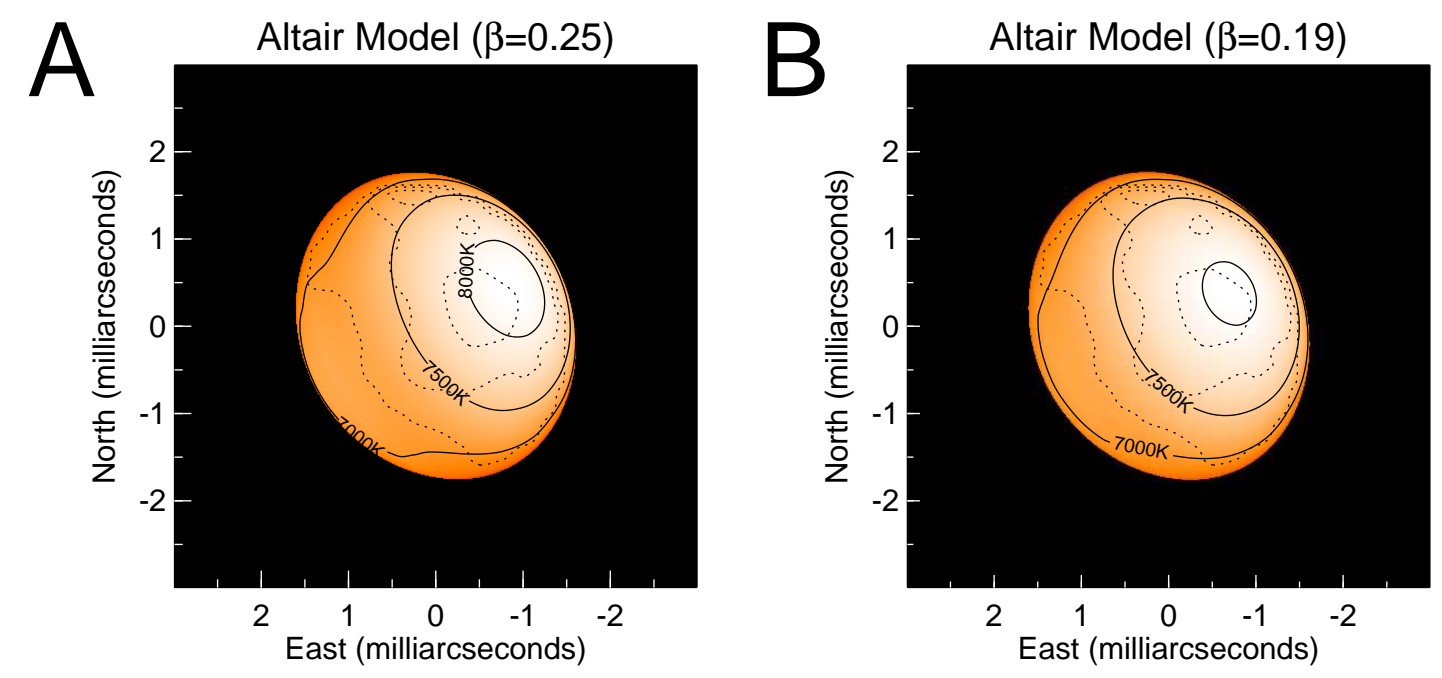

Figure 3: The panels show synthetic images of Altair $(\lambda=1.65 \mu \mathrm{m})$ adopting conventional rapid rotation models. A) is the best-fit model assuming standard gravity-darkening coefficient for radiative envelopes $\left(\beta=0.25, \chi_{\nu}^{2}=\right.$ $1.79)$ while $\mathrm{B})$ shows the result when $\beta$ is a free parameter $\left(\beta=0.190, \chi_{\nu}^{2}=1.37\right)$. For both panels, the specific intensities at $1.65 \mu \mathrm{m}$ were converted into the corresponding blackbody temperatures and contours for $7000 \mathrm{~K}$, $7500 \mathrm{~K}$, and $8000 \mathrm{~K}$ are shown. We have overplotted the contours from the CHARA image (Figure 2A) as dotted lines to facilitiate intercomparison. 
Table 1: Best-fit parameters for Roche - von Zeipel models of Altair. Parameter descriptions: Inclination $\left(0^{\circ}\right.$ is pole-on, $90^{\circ}$ is edge-on) \& Position Angle (degrees East of North) describe our viewing angle, $T_{\text {pole }} / \mathrm{R}_{\text {pole }}$ describe the temperature and radii of the pole (alternatively, one can describe the temperature and radii at the equator $\left.T_{\text {eq }} / R_{\text {eq }}\right), \omega$ is the angular rotation rate as a fraction of critical breakup rate, $\beta$ is the gravity-darkening coefficient. Models assumed stellar mass $1.791 \mathrm{M}_{\odot}([15)$, metallicity $[\mathrm{Fe} / \mathrm{H}]=-0.2(32)$, and distance $5.14 \mathrm{pc}(33)$.

\begin{tabular}{lll} 
Parameters & $\beta$ Fixed & $\beta$ Free \\
\hline Inclination (degs) & $62.7 \pm 1.5$ & $57.2 \pm 1.9$ \\
Position Angle (degs) & $-61.7 \pm 0.9$ & $-61.8 \pm 0.8$ \\
$\mathrm{~T}_{\text {pole }}(\mathrm{K})$ & $8710 \pm 160$ & $8450 \pm 140$ \\
$\mathrm{R}_{\text {pole }}\left(\mathrm{R}_{\odot}\right)$ & $1.661 \pm 0.004$ & $1.634 \pm 0.011$ \\
$\quad(\mathrm{mas})$ & $1.503 \pm 0.004$ & $1.479 \pm 0.010$ \\
$\mathrm{~T}_{\text {eq }}(\mathrm{K})$ & $6850 \pm 120$ & $6860 \pm 150$ \\
$\mathrm{R}_{\text {eq }}\left(\mathrm{R}_{\odot}\right)$ & $2.022 \pm 0.009$ & $2.029 \pm 0.007$ \\
$\quad($ mas $)$ & $1.830 \pm 0.008$ & $1.835 \pm 0.007$ \\
$\omega \quad$ & $0.902 \pm 0.005$ & $0.923 \pm 0.006$ \\
$\beta \quad$ & 0.25 (Fixed) & $0.190 \pm 0.012$ \\
Model V Mag & 0.765 & 0.765 \\
Model H Mag & 0.225 & 0.220 \\
Model v sin $i(\mathrm{~km} / \mathrm{s})$ & 241 & 240 \\
\hline Reduced $\chi^{2}:$ & & \\
$\quad$ Total & 1.79 & 1.37 \\
$\quad$ Closure Phase & 2.08 & 1.73 \\
$\quad$ Vis & & 1.10 \\
$\quad$ Triple Amp & 1.48 & 1.58 \\
\hline
\end{tabular}




\section{Supplemental Online Materials}

The Michigan Infrared Combiner (MIRC) is a new instrument on the Georgia State University CHARA interferometric array; MIRC and the CHARA Array have both been described in detail (31,21) and MIRC commissioning results have already been presented (34). Here in the supplemental online materials, we present validation studies of our data pipeline and image reconstruction methods.

MIRC is an image plane combiner which currently combines light from four CHARA telescopes simultaneously. The four CHARA beams are filtered by single-mode fibers and the beams are rearranged into a 1-dimensional non-redundant pattern and brought to a focus. These overlapping beams create six interference fringes, each with a unique spatial frequency. The pattern is then focused by a cylindrical lens into a "line" of fringes which are subsequently dispersed by a simple spectrograph with spectral resolution $\Delta \lambda \sim 0.035 \mu \mathrm{m}$. Fast readout of the Rockwell PICNIC camera (frame time $5.5 \mathrm{~ms}$ ) effectively freezes the atmosphere under most seeing conditions in the infrared. In this way, MIRC can measure 6 visibilities, 4 closure phases, and 4 triple amplitudes simultaneously over 8 spectral channels spanning the astronomical Hband $(\lambda=1.50-1.74 \mu \mathrm{m})$.

Here we briefly outline the MIRC data analysis method. After background subtraction, the fringe patterns are analyzed by taking the Fourier transform. From this intermediate data, the fringe phases and amplitudes can be combined to form the triple product, often expressed as complex number that can be coherently averaged (the angle argument is the closure phase) (5). The power spectra are also accumulated for visibility-squared estimation. Bias in the power spectrum is subtracted using a combination of "foreground" observations (data taken with halted delay lines) and measurements using high spatial frequencies immune to contamination by true fringe power. 
The above procedures result in tabulations of uncalibrated squared-visibilities and (complex) triple amplitudes. In order to calibrate the amplitudes of these quantities, we must estimate how much light is injected into the fibers during fringe measurements. We use spinning choppers to partially obscure each input pupil during fringe acquisition, chopping each beam at a unique frequency $(25 \mathrm{~Hz}, 30 \mathrm{~Hz}, 35 \mathrm{~Hz}, 40 \mathrm{~Hz})$. Since fringes are spatially-modulated, we can use the temporally-chopped intensities to obtain an estimate of the fiber coupling efficiencies simultaneous with fringe measurements.

At this stage in the analysis, individual data files have been calibrated but no estimates of the system visibilities have been made. Since we have a single-mode fiber system, the system visibilities are highly stable, however the image-plane combiner is susceptible to temporal decoherence since the $5.5 \mathrm{~ms}$ exposure time is not short enough to completely freeze turbulence. We track these and any other changes in system visibility by observing calibrator objects with known sizes, in this case $\gamma$ Lyr and $v$ Peg with estimated uniform disk diameters of $0.74 \pm 0.10$ mas $(35)$ and $1.05 \pm 0.05$ mas (MIRC/CHARA) respectively. While uncertainties in calibrator diameters are often the dominant error for long baseline interferometers like the CHARA Array, such errors are generally not important for Altair which is unusually highlyresolved - our dominant errors are from imprecise calibration of mean fiber coupling and fast changes in atmospheric coherence time.

In order to validate our pipeline and imaging procedures, we carried out observations of the binary $\iota$ Peg. This binary is well-suited for calibration, having been observed by IOTA, NPOI, PTI, and MIRC/CHARA. Using $\iota$ Peg we have calibrated our "closure phase sign" which removes the $180^{\circ}$ ambiguity in imaging. Furthermore, we have confirmed our wavelength calibration at the $0.3 \%$ level through comparison with previously published and new PTI measurements (36). The top panels of Figure S1 show the snapshot Fourier coverage of our $\iota$ Peg observations from UT2006Sep02 as well as our calibrated visibility-squared data compared to 
the binary model fit.

$\iota$ Peg represented a suitable target for validation of the MACIM imaging algorithm (23) as well. The bottom panels of Figure S1 show the MACIM image reconstruction of the $\iota$ Peg binary along with the best-fit binary model; these results are consistent with the prediction from the orbit (36). The MACIM image is in excellent agreement with the model, including the size determinations of the two stars. We also show the imaging results using the CLEAN algorithm (25) with self-calibration. A detailed analysis of $\iota$ Peg will follow in a subsequent paper.

Since imaging with optical interferometry is still novel, we present here the calculated interferometric observables from our MACIM/MEM image presented in Figure 2 of the mainn report. Figures S2-4 contain all the individual data points used in this Report and they are compared with the imaging results. As found in other studies (37,34), the closure phase quantities are particularly robust and precise, showing none of the calibration difficulties typically encountered for measurements of visibility amplitudes. The calibrated data for Altair, stored in the Optical Interferometry data exchange format (OI-FITS) (38), are available from the authors.

The final topic to discuss is the special imaging procedure for Altair. Firstly, we emphasize that use of MEM for interferometric imaging is standard practice (24, 39) and the specific program MACIM (23) has been validated on other test data (40). Hence, we will not explain the fundamentals of interferometric imaging here or why specialized software is required for optical interferometers (38), but rather refer the reader to the extensive literature (41, 42, 43, 44, 37).

For imaging, we treated each wavelength channel as providing a distinct set of $(\mathrm{u}, \mathrm{v})$ plane coverage, ignoring any wavelength-dependence of the image itself - this procedure is sometimes referred to as wavelength-super-synthesis. This assumption is well-justified for infrared intensities of hot stars since the relative intensities across the photosphere for the Altair model are nearly identical at $1.5 \mu \mathrm{m}, 1.65 \mu \mathrm{m}$, and $1.8 \mu \mathrm{m}$, showing relative distortions of $<0.5 \%$. This 
level of inaccuraciy is much less than our observed temperature reconstruction errors of $4 \%$. Note that our von Zeipel modeling code did treat this wavelength-dependence better by splitting the $\mathrm{H}$ band into 4-different sub-bands for fitting to the wavelength-dependent visibility and closure phases data.

The main difficulty in imaging the surface of a star is that the photospheric emission is expected to show a sharp fall-off at the limb. In terms of Fourier modes, this sharp cutoff is encoded in very long baseline visibilities which can not be observed. This in combination with the MEM procedure causes extensive "spreading out" in a reconstructed image, with more spreading happening where we lack the longest baseline data. From this perspective, we identify contradictory goals for the imaging procedure - smooth out the image as much as possible except right at the edge where we expect the sharp cutoff in emission. This problem is similar to that encountered by others (45) attempting to image diffuse circumstellar material surrounding an unresolved point source. In the latter case, the imaging procedure was stabilized by using a point-source model as an "image prior" to the MEM procedure, based on a priori knowledge of the target under scrutiny.

For the imaging reported here, we used a uniform ellipse as a prior to the MACIM/MEM imaging. For the given elliptical prior, we ran the MACIM/MEM algorithm and found the image with maximum entropy fitting the data with a $\chi_{\nu}^{2} \sim 1$. This procedure was robust - the MEM prior naturally limited the flux inside the elliptical boundary while the Maximum Entropy maximization tended to spread out the flux as much as possible consistent with the data itself.

The main complication in applying the above procedure is that we do not know a priori which ellipse to choose for our MEM prior. One could use the uniform ellipse derived from short baseline data, e.g., from the PTI data of Van Belle et al. (12); however, one realizes that this is not optimum since the best-fit uniform ellipse underestimates the actually photospheric boundary of an oblate star with gravity darkening (in the case of Altair by $~ 5 \%$ ). In order to 
keep our imaging procedure general and avoid a bias through our choice of one specific elliptical prior, we carried out MACIM/MEM imaging on a grid of 500 different uniform ellipses spanning a range of possible sizes, elongations, and position angles. As expected, the "entropy" of the final image varied depending on the prior we adopted and it was a simple matter to find the global Maximum Entropy image from the ensemble.

As a final check on our calibration consistency, we carried out the above imaging procedures on the MIRC/CHARA data split by observing night. Figure S5 shows the final MACIM/MEM images for the two nights separately. Based on the variation between the two independent images, we estimate the photometric uncertainty in the final reconstruction to correspond to $\pm 4 \%$ in intensity across the photosphere (with a worst case $\pm 10 \%$ - near the limb of the star). The high degree of similarity gives us confidence that the final image reconstruction is not corrupted by night-to-night calibration errors.

Lastly, we comment on some confusion in the literature. Unfortunately the first published results on Altair (12) inadvertently had the (u,v) coordinates switched. This mistake was compounded in the next paper on Altair, Ohishi et al. (16) from NPOI, which also made a coordinate mistake. These errors were noticed by Domiciano de Souza (46) who attempted a correction in order to combine all the data together in a self-consistent way (although this was not mentioned in the paper itself). Most recently, Peterson et al. (15) re-analyzed the original NPOI data, correcting the UV coordinate mistakes and pointing out the original PTI errors. However, this paper appears to have gotten the closure phase calibration incorrect - causing a $180^{\circ}$ rotation in their published synthetic model images. In most cases, these errors affected only the inferred viewing orientation of Altair, thus they did not impact the astrophysical interpretation of the Altair data. 

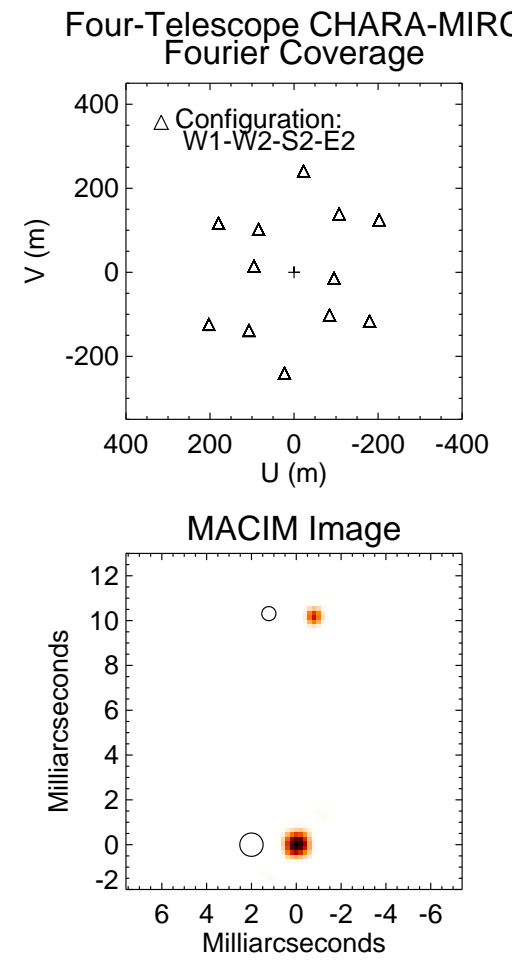
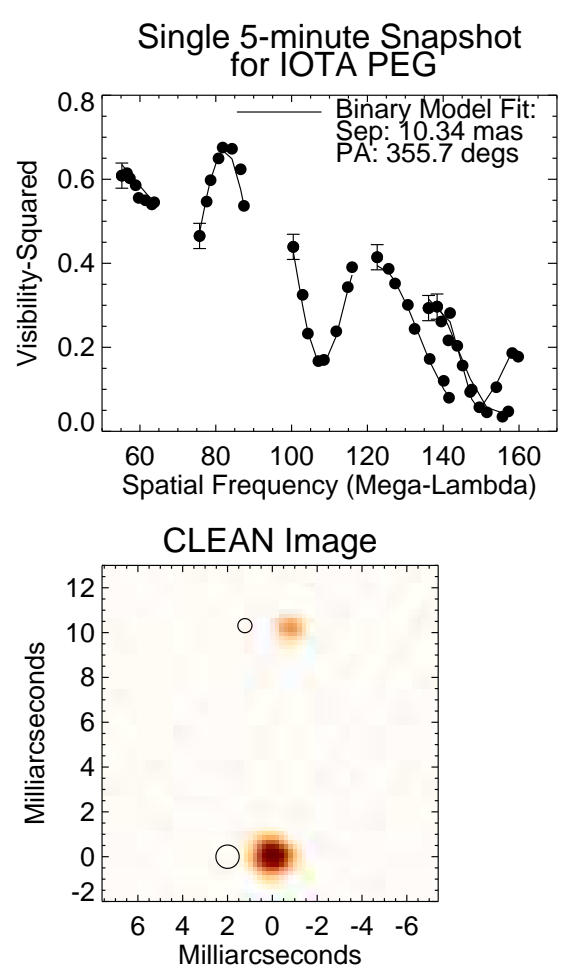

Figure 4: These four panels validate the MIRC/CHARA pipeline and the MACIM image reconstruction software. The top left panel shows the snapshot 4-telescope Fourier coverage for an observation of the calibration binary $\iota$ Peg on UT 2006 Sep 02 . The top right panel shows the calibrated squared-visibility data along with the best-fit binary model (representative errors are shown only for long-wavelength channel for clarity). The bottom panels show a comparison of the image reconstructions using the MACIM and CLEAN algorithms with the best-fit binary model (circles offset 2 mas to the east). 


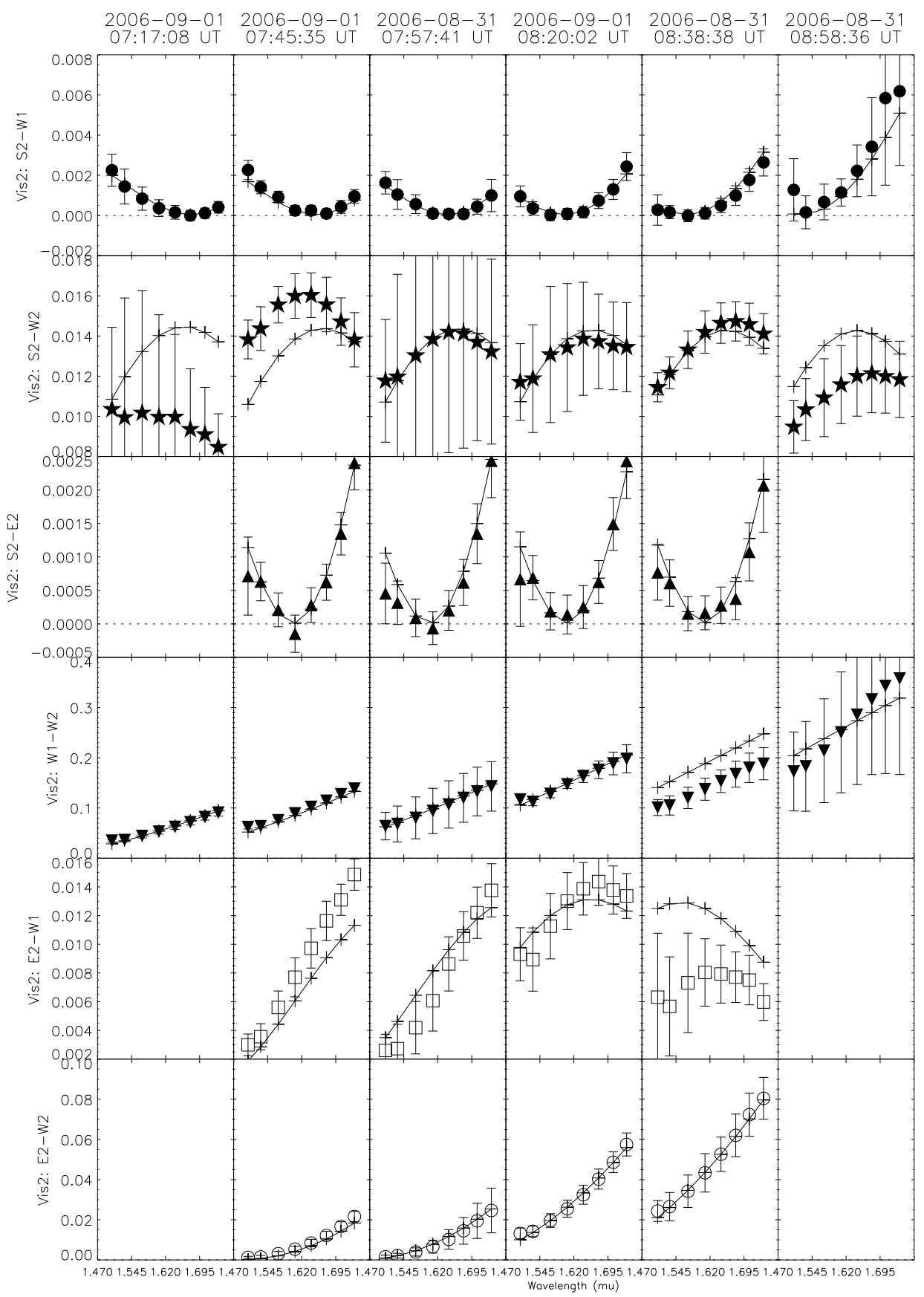

Figure 5: This figure shows the squared-visibilities (with errors) observed for Altair along with the calculated values from the MACIM/MEM image (line with crosses) presented in left panel of Figure 2. Each column is a different observing time while each row represents a different baseline. Inside each panel, the x-axis shows the wavelength of the spectrometer channels. Note that the visibility nulls shown above for baselines S2-W1 and S2-E2 are second nulls while the visibilities in E2-W2 and E2-W1 baselines are slightly before the first null. 


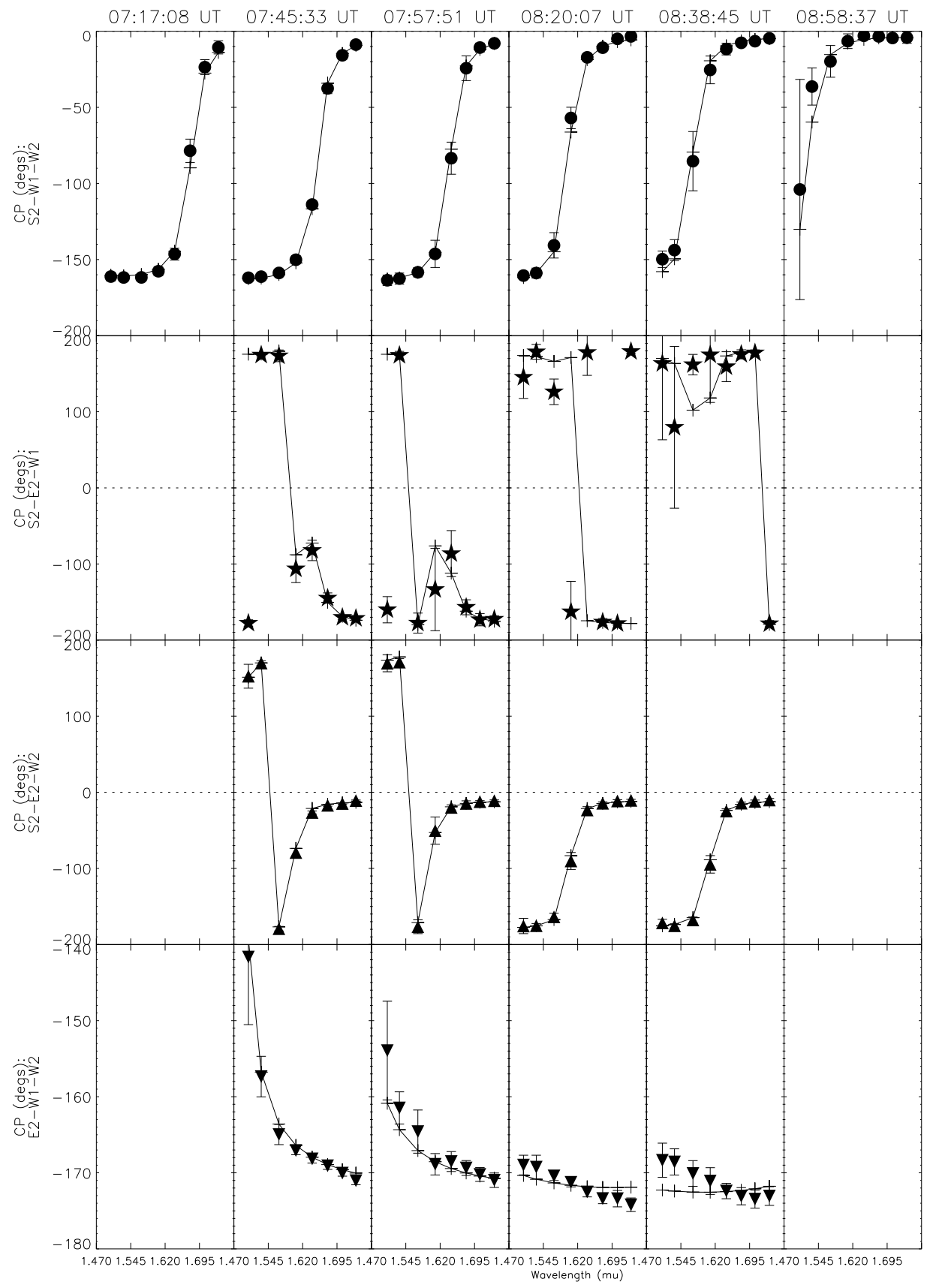

Figure 6: All closure phase measurements are shown for the Altair observations along with results from MACIM/MEM image (line with crosses). Note that the closure phase has a $360^{\circ}$ phase ambiguity, thus a phase of $+180^{\circ}$ and $-180^{\circ}$ are identical in the panels above. The columns are different times and the rows represent different closure triangles. 


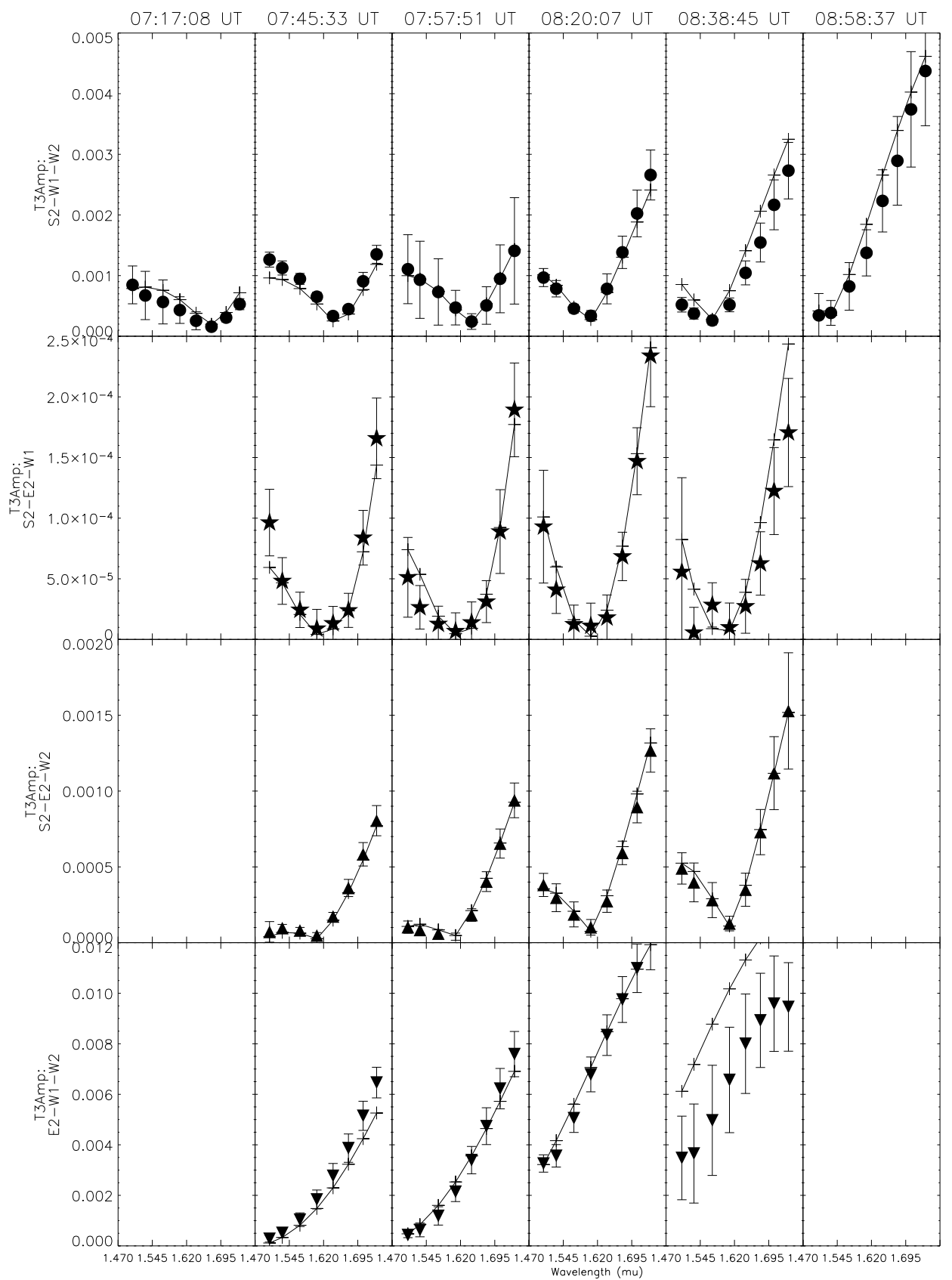

Figure 7: All triple amplitude measurements are shown for the Altair observations along with results from MACIM/MEM image (line with crosses). The columns are different times and the rows represent different closure triangles. 

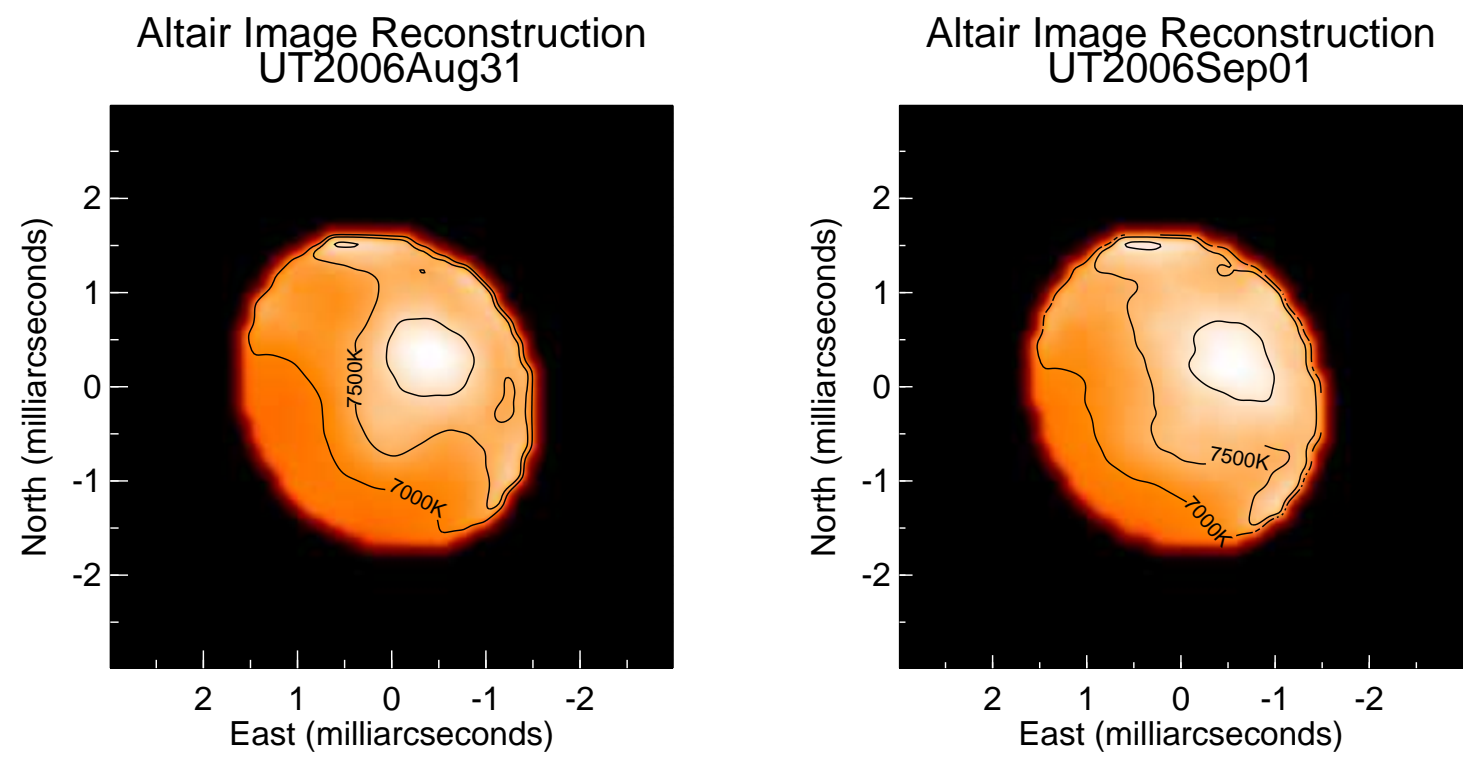

Figure 8: Similar to Figure 2 of main Report, except here we compare imaging results from two independent data sets. These image reconstructions agree at the $4 \%$ level rms, with maximum deviations of $10 \%$ near the limb.

\section{References and Notes}

1. A. Maeder, G. Meynet, ARA\&A 38, 143 (2000).

2. J. D. Landstreet, A\&A Rev. 4, 35 (1992).

3. P. Kervella, A. Domiciano de Souza, A\&A 453, 1059 (2006).

4. P. Barai, et al., ApJ 608, 989 (2004).

5. J. D. Monnier, Reports of Progress in Physics 66, 789 (2003).

6. H. A. Abt, N. I. Morrell, ApJS 99, 135 (1995).

7. H. A. Abt, H. Levato, M. Grosso, ApJ 573, 359 (2002). 
8. M. Pinsonneault, ARA\&A 35, 557 (1997).

9. G. E. Brown, et al., New Astronomy 5, 191 (2000).

10. N. Gehrels, et al., Nature 437, 851 (2005).

11. H. von Zeipel, MNRAS 84, 684 (1924).

12. G. T. van Belle, D. R. Ciardi, R. R. Thompson, R. L. Akeson, E. A. Lada, ApJ 559, 1155 (2001).

13. D. M. Peterson, et al., Nature 440, 896 (2006).

14. J. P. Aufdenberg, et al., ApJ 645, 664 (2006).

15. D. M. Peterson, et al., ApJ 636, 1087 (2006).

16. N. Ohishi, T. E. Nordgren, D. J. Hutter, ApJ 612, 463 (2004).

17. A. Domiciano de Souza, et al., A\&A 407, L47 (2003).

18. H. A. McAlister, et al., ApJ 628, 439 (2005).

19. G. T. van Belle, et al., ApJ 637, 494 (2006).

20. S. Jackson, K. B. MacGregor, A. Skumanich, ApJ 606, 1196 (2004).

21. J. D. Monnier, J.-P. Berger, R. Millan-Gabet, T. A. Ten Brummelaar, SPIE v.5491. (ed. Traub), W. A. Traub, ed. (2004), pp. 1370-+.

22. Online Supplemental Materials.

23. M. J. Ireland, J. D. Monnier, N. Thureau, SPIE v.6268 (eds. Monnier, Schöller, Danchi) (2006). 
24. R. Narayan, R. Nityananda, ARA\&A 24, 127 (1986).

25. J. A. Högbom, A\&AS 15, 417 (1974).

26. R. Kurucz, ATLAS9 Stellar Atmosphere Programs and 2 km/s grid. 13 (1993).

27. S. Jackson, K. B. MacGregor, A. Skumanich, ApJS 156, 245 (2005).

28. A. Claret, A\&A 359, 289 (2000).

29. F. Espinosa Lara, M. Rieutord, astro-ph/0702255 (2007).

30. C. C. Lovekin, R. G. Deupree, C. I. Short, ApJ 643, 460 (2006).

31. T. A. ten Brummelaar, et al., ApJ 628, 453 (2005).

32. D. Erspamer, P. North, A\&A 398, 1121 (2003).

33. M. A. C. Perryman, ESA, The HIPPARCOS and TYCHO catalogues. (ESA Publications Division, ESA SP Series vol no: 1200, 1997).

34. J. D. Monnier, et al., SPIE v.6268 (eds. Monnier, Schöller Danchi) (2006).

35. S. K. Leggett, et al., A\&A 159, 217 (1986).

36. A. F. Boden, et al., ApJ 515, 356 (1999).

37. J. D. Monnier, et al., ApJ 602, L57 (2004).

38. T. A. Pauls, J. S. Young, W. D. Cotton, J. D. Monnier, PASP 117, 1255 (2005).

39. P. G. Tuthill, J. D. Monnier, W. C. Danchi, E. H. Wishnow, C. A. Haniff, PASP 112, 555 (2000).

40. P. R. Lawson, et al., SPIE v.6268 (eds. Monnier, Schöller, Danchi) (2006). 
41. T. J. Cornwell, P. N. Wilkinson, MNRAS 196, 1067 (1981).

42. T. J. Pearson, A. C. S. Readhead, ARA\&A 22, 97 (1984).

43. J. E. Baldwin, et al., A\&A 306, L13+ (1996).

44. C. A. Hummel, et al., AJ 125, 2630 (2003).

45. J. D. Monnier, et al., ApJ 605, 436 (2004).

46. A. Domiciano de Souza, et al., A\&A 442, 567 (2005).

47. We acknowledge contributions from Ajay Tannirkulam, Scott Webster, Andy Boden, Bob Zavala, Chris Tycner, Christian Hummel, Deane Peterson, Jason Aufdenberg, PJ Goldfinger, and Steve Golden. Research at the CHARA Array is supported by the National Science Foundation through grants AST 06-06958 and AST 03-52723 and by the Georgia State University through the offices of the Dean of the College of the Arts and Sciences and the Vice President for Research. 